\title{
Article \\ State of Health Prediction of Power Connectors by Analyzing the Degradation Trajectory of the Electrical Resistance
}

\author{
Jimmy Martínez ${ }^{1}\left(\mathbb{D}\right.$, Jordi-Roger Riba ${ }^{1, *(\mathbb{D})}$ and Manuel Moreno-Eguilaz ${ }^{2}(\mathbb{0})$ \\ 1 Electrical Engineering Department, Universitat Politècnica de Catalunya, 08222 Terrassa, Spain; \\ jimmy.arturo.martinez@upc.edu \\ 2 Electronics Engineering Department, Universitat Politècnica de Catalunya, 08222 Terrassa, Spain; \\ manuel.moreno.eguilaz@upc.edu \\ * Correspondence: jordi.riba-ruiz@upc.edu
}

check for

updates

Citation: Martínez, J.; Riba, J.-R.; Moreno-Eguilaz, M. State of Health Prediction of Power Connectors by Analyzing the Degradation Trajectory of the Electrical Resistance. Electronics 2021, 10, 1409. https://doi.org/ 10.3390/electronics10121409

Academic Editors: Zehui Mao,

Xinggang Yan, Hamed Badihi and Davide Astolfi

Received: 7 May 2021

Accepted: 9 June 2021

Published: 11 June 2021

Publisher's Note: MDPI stays neutral with regard to jurisdictional claims in published maps and institutional affiliations.

Copyright: (c) 2021 by the authors. Licensee MDPI, Basel, Switzerland. This article is an open access article distributed under the terms and conditions of the Creative Commons Attribution (CC BY) license (https:// creativecommons.org/licenses/by/ $4.0 /)$.

\begin{abstract}
Estimating the remaining useful life (RUL) or the state of health (SoH) of electrical components such as power connectors is still a challenging and complex task. Power connectors play a critical role in medium- and high-voltage power networks, their failure leading to important consequences such as power outages, unscheduled downtimes, safety hazards or important economic losses. Online condition monitoring strategies allow developing improved predictive maintenance plans. Due to the development of low-cost sensors and electronic communication systems compatible with Internet of Things (IoT) applications, several methods for online and offline SoH determination of diverse power devices are emerging. This paper presents, analyzes and compares the performance of three simple and effective methods for online determination of the $\mathrm{SoH}$ of power connectors with low computational requirements. The proposed approaches are based on monitoring the evolution of the connectors' electrical resistance, which defines the degradation trajectory because the electrical resistance is a reliable indicator or signature of the $\mathrm{SoH}$ of the connectors. The methods analyzed in this paper are validated by means of experimental ageing tests emulating real degradation conditions. Laboratory results prove the suitability and feasibility of the proposed approach, which could be applied to other power products and apparatus.
\end{abstract}

Keywords: electrical connectors; state of health; condition monitoring; parameter identification; predictive maintenance

\section{Introduction}

Power connectors installed in medium- and high-voltage power lines are usually placed in critical points of the power grid, being critical elements to ensure a reliable power dispatch. Probably because of their abundance, low cost and simplicity, they often are not being paid the attention they deserve, despite their key role in the reliability and availability of the power grid. Sudden power connector failure could induce important power outages with expensive and damaging consequences [1]. It is difficult to find continuity of supply data because there are differences in the type of interruptions monitored, indicators used and data collection procedures applied in different countries. According to the Council of European Energy Regulators, the minutes lost per year in European countries due to planned interruptions range from $10 \mathrm{~min}$ to $500 \mathrm{~min}$, whereas for unplanned interruptions, they range from $10 \mathrm{~min}$ to $1100 \mathrm{~min}$ [2]. Operators of power systems work hard to offer a continuous, safe and reliable power delivery to their customers, thus trying to minimize the number and the effects of service outages [3]. The application of predictive maintenance strategies based on the prediction or diagnosis of the state of health $(\mathrm{SoH})$ allows achieving this goal.

The electrical resistance has been effectively used as an indicator of the SoH of electrical connectors [4,5]. It is known that the natural ageing process of the connector increases the electrical resistance along its lifetime [6], thus increasing its operating temperature and 
tending to overheat the connector. This temperature increment, in turn, raises the electrical resistance, thus affecting the electrical and thermal behaviors of the connector [7].

It is known that electrical resistance is characterized by two components, i.e., contact and bulk resistance [8]. The bulk resistance component is almost defined by the geometry of the connector and the electrical resistivity of the constitutive materials. The contact resistance component includes two terms, the film and constriction resistance [9]. It depends upon several inner factors (structure, connection material or surface topology), environment conditions (temperature, humidity or vibration), and working load (supply frequency or current) [10]. Two predominant ageing processes affect the long-term performance of power connectors, i.e., contact pressure and chemical reactions generated at the matting interfaces. The contact resistance tends to increase with the cyclic pressure variations due to the daily peak and off-peak load patterns, as well as due to poor installation practices. Chemical reactions at the contact interface generate non-conductive compounds, which negatively affect the contact resistance [11]. Due to its complexity, an exhaustive analysis of the time evolution of the contact resistance is a difficult task [12].

Diagnosis of electrical and electronic systems has received much attention over the last few decades [13]. Power lines and electrical substations are inspected periodically to determine their condition. However, due to the lack of online data, at present, the most applied inspection systems include manual, robot and unmanned aerial vehicle inspection [14].

Condition monitoring is directly related to different methods for identifying changes occurring in a system due to the development of faults or the degradation of the $\mathrm{SoH}$, thus generating an alarm to indicate a possible failure or degradation of the $\mathrm{SoH}$ [15]. On-line condition monitoring is an active field of research in power systems [16-19]. To apply effective predictive maintenance strategies and to reduce maintenance and unexpected outages and shutdown costs, there is an imperious need to detect anomalous or degraded behavior modes in the early stage, when the degraded behavior is still developing. However, detecting abnormal behaviors at the early stage is not an easy subject, as slight changes are often difficult to diagnose, so great care has to be taken to minimize false alarm events [20].

Real-time data acquisition and the associated deployment of distributed sensors is a key point for the expansion of intelligent power systems. Such systems allow a more stable and controllable power delivery since real-time data allow applying different condition monitoring strategies [21]. Therefore, the online measurement of the electrical resistance using electronic devices is a key point for continuous monitoring of the electrical resistance of the connectors in order to develop effective SoH prediction tools. Voltage, current and temperature sensors can be used for nondestructive detection, location and diagnosis of faults in power systems. Traditionally, these sensors have been applied to diagnose the faults after their occurrence. Despite improvements in the system's robustness, failures cannot be completely eliminated, and they are somewhat unpredictable, so maintenance operations are required before failure [22]. There is a growing demand to develop prognostic methods to predict the faults in advance, i.e., when the system is fully operational, before major faults occurrence. This strategy allows operators to estimate the residual lifetime and to schedule predictive maintenance operations [22]. In addition, suitable real-time analysis algorithms are key elements for this purpose [23].

Although statistical methods have been widely used, they are not the best choice to tackle fault diagnosis problems because such methods estimate probability distributions based on large numbers of training samples [24], which often are not available; thus, this strategy is costly and time-consuming. Different strategies can be applied to determine the $\mathrm{SoH}$, including approaches based on physical-mathematical models, data-driven algorithms or hybrid approaches combining mathematical models and data-driven methods $[25,26]$. These last methods can perform better since they potentially combine the benefits of the two other approaches [5]. Physical-mathematical models rely on a mathematical description of the physics or phenomena determining the degradation process, thus requiring little historical data. Contrarily, data-driven approaches predict the behavior 
of the system under analysis from historical data [26] collected using different sensors and applying appropriate signal processing algorithms [20]. Data-driven methods include different strategies, which can be based on statistics, time-series or artificial intelligence algorithms.

SoH and RUL estimation are currently hot research topics. A review of lithium-ion batteries prognosis methods is presented in [27], where two main groups of models are described, i.e., model-based methods (they establish a degradation model based on a physicochemical description of the problem or on empirical correlations in large amounts of data) and data-driven methods (they do not use a physics model) approaches. In [28], a data-driven approach based on machine learning was proposed to predict battery cycle life because it is suitable to predict the dynamic behavior of complex systems. In [29], the finite element method was applied to estimate the fatigue life of the gas turbine blade.

There is an imperious need to develop effective SoH strategies. However, there is a lack of research works dealing with this topic for power connectors. It could be attributed to the fact that power connectors are considered simple elements, despite the critical role they play in power applications, and because they are still not instrumented, i.e., there is no electrical data available to monitor connectors' performance.

This paper proposes predicting the SoH of power connectors by studying the degradation trajectory of the electrical resistance because it is a reliable indicator of power connectors' performance [30,31]. Approaches based on the study of the degradation trajectory are gaining consideration [32], but much work remains to be carried out in this area.

There is a scarcity of works related to the online SoH diagnosis of power connectors, this work presenting and assessing three simple alternatives with a reduced computational burden. The first method (linear fitting SoH or LF-SoH) predicts the SoH of each individual connector by comparing the last measured values of the resistance against the predictions determined by a least-squares linear regression model. The remaining two methods evaluated in this work are based on a non-linear model of the degradation trajectory of the contact resistance based on the Braunovic equation [33], instead of assuming a linear degradation model. The second method (non-linear fitting SoH or NLF-SoH) directly determines the $\mathrm{SoH}$ by comparing the last measured values of the resistance against the predictions determined by a least-squares fitting of the Braunovic model [33], while the third one applies the Markov chain Monte Carlo (MCMC) method [34] for this purpose, so it is called MCMC-NLF-SoH. It is worth noting that the three methods compared in this work focus on each specific connector, being adapted to the particular characteristics of each power device, only requiring the past and current values of the electrical resistance to determine its SoH. The behavior of the analyzed methods is assessed by means of experimental data obtained from accelerated degradation tests (ADTs) since they are designed to analyze the long-term performance of power connectors by minimizing the testing time [35] because due to their long lifetimes, it is not practical to acquire degradation data in an acceptable time. By measuring the voltage drop between the connectors' terminals, the electrical current and the operating temperature, the degradation trajectory of the electrical resistance is continuously monitored, which is the base to predict the SoH.

Recently, different projects in line with the Internet of Things (IoT) are being developed for high-voltage equipment. They include different functions such as self-diagnosis, wireless communications [36], the use of different sensors [4,37] and energy harvesting units.

Electrical substations are usually inspected visually or using thermal and/or ultraviolet cameras, thus applying preventive maintenance strategies. These tasks require specialized technicians and scheduled inspections, thus resulting in expensive and difficult to apply under adverse weather conditions. Smart connects will allow shifting from preventive to predictive maintenance. However, the implementation of smart connectors in a real high-voltage environment is not fully developed, being a challenging task due to 
the complex systems involved, including energy harvesting, sensing systems and wireless communications.

The contributions of this paper are as follows. First, it contributes to develop and test methods with low computational requirements for an online SoH prediction of electrical connectors from experimental data. This is an area with a clear lack of research works. Second, the methods here analyzed are appealing because of their simplicity and fast response, thus being compatible with low-cost microcontrollers that soon will integrate the new generations of smart connectors.

Third, the solution proposed in this paper is in line with the development of smart grids, digital substations and the Internet of Things (IoT), where predictive maintenance, prediction of the remaining useful life and the $\mathrm{SoH}$ are trending topics. However, installed power connectors do not include these developments, this paper making a clear development in this field. Fourth, the strategy exposed in this work adapts to the particular behavior and evolution of each connector since it is known that there is huge variability among connectors. This approach is able to anticipate severe faults, thus allowing to control and limit connectors' degradation process while enabling to apply predictive maintenance plans. Finally, the methods described in this paper present reduced computational requirements, being possible to be applied in real-time and can be easily adapted to determine the SoH of many other power devices.

\section{The Analyzed Connectors}

This work analyzes ICAU120 medium voltage compression connectors (SBI Connectors, Sant Esteve Sesrovires, Spain), which are mostly applied in alternating current (AC) medium-voltage systems. Compression connectors are usually applied in medium-voltage systems because they offer reduced electrical resistance and a stable connection. These bimetallic connectors are made of copper are aluminum, the aluminum and copper parts being connected by friction welding. The connectors are connected to $120 \mathrm{~mm}^{2}$ aluminum conductors by compression, using a hexagonal crimping machine and applying conductive grease that is able to operate up to $140{ }^{\circ} \mathrm{C}$.

This work analyzes medium voltage connectors, although the final goal is to extend the results of this study to substation connectors. Medium voltage connectors are analyzed because they have a shorter lifetime and lower current and power ratings compared to that of substation connectors, thus simplifying the requirements of the tests, testing time, and associated costs.

\section{The Electrical Resistance of the Connectors}

As the connector resistance increases, more power losses and heat are generated, accelerating the degradation of the interface between the conductor and the connector and thus increasing its overall electrical resistance. This is why the SoH is focused on the degradation trajectory of the electrical resistance with time. SoH strategies require an easy-to-apply end-of-life (EOL) criterion, which is set to $1.4 \cdot R_{0}[5]$, where $R_{0}$ is the initial value of the connector resistance, i.e., the value measured during installation of the connector. However, there are no methods for a direct online measurement of the electrical resistance, so an indirect measurement is required. It is important to note that the expected value of the electrical resistance is of some micro-Ohms, so the measurement of the electrical resistance under alternating current supply is a challenging task.

Due to the temperature dependence of the connector resistance, it is usually referred to as $20{ }^{\circ} \mathrm{C}$. The instantaneous value of the resistance of the connector referred to $20{ }^{\circ} \mathrm{C}$, $R_{20}{ }^{\circ} \mathrm{C}(t), t$ being the time, is calculated from the acquisitions of the voltage drop $\Delta V_{R M S}$, the current $I_{R M S}$ and $\varphi$ as in Equation (1), where $\varphi$ is the phase shift between the voltage drop and the current and $\alpha$ is the temperature coefficient of the resistance, which value is $0.004{ }^{\circ} \mathrm{C}^{-1}$ for aluminum. It is noted that the voltage drop and current in Equation (1) are 
calculated in the root mean square (RMS) value, and these values changing slowly with time.

$$
R_{20}{ }^{\circ} \mathrm{C}(t)=\frac{\Delta V_{R M S}(t) \times \cos \varphi}{I_{R M S}(t) \times[1+\alpha \times(T(t)-20)]}
$$

\section{The Applied Heat Cycle Tests (HCTs) and the Equipment Involved}

As explained, ADTs are commonly applied to obtain degradation data defining the behavior of the studied system in a fast manner. To this end, HCTs were performed in the high-current laboratory of the Universitat Politècnica de Catalunya according to the requirements of the IEC 61238-1-3:2018 standard [11]. It is noted that data acquired from the HCTs are used to simulate the online acquired data from a real application.

HCTs were performed using an electrical loop consisting of seven ICAU120 bimetallic connectors joined to a $120 \mathrm{~mm}^{2}$ aluminum conductor. Figure 1 a shows the experimental loop and the connectors. The voltage drop across the terminal points of the seven connectors using wire equalizers (see Figure 1b), the current in the loop and the temperature of each connector were measured to determine the electrical resistance of all connectors.

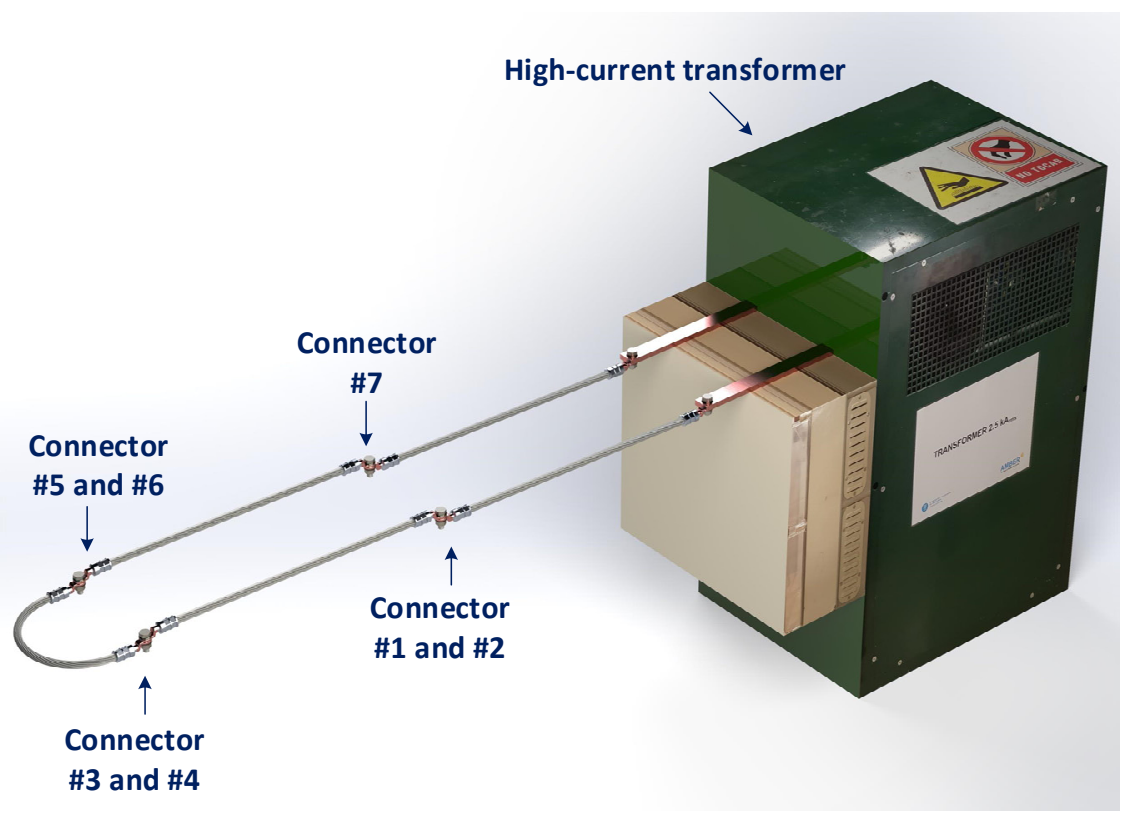

(a)

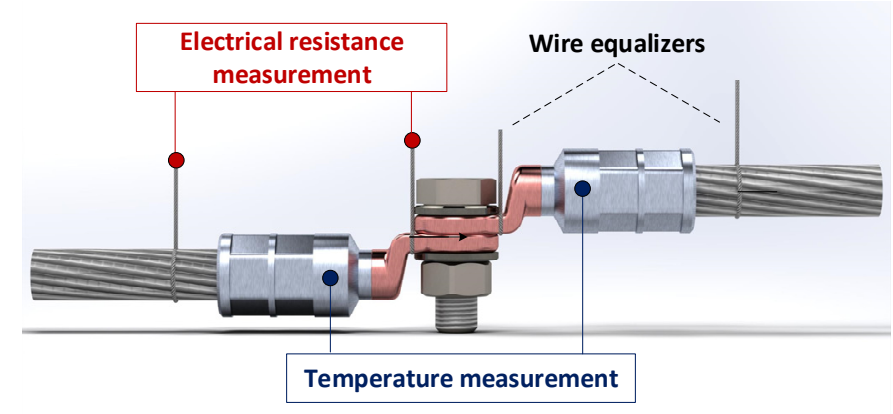

(b)

Figure 1. (a) The electrical loop used in heat cycle tests. (b) The contact resistance measurement of the ICAU120 bimetallic connectors.

To accelerate the natural ageing of the connectors, around 140 HCTs were run for about $92 \mathrm{~h}$. Heat cycles consist of two phases, namely heating and cooling phases. The consecutive heating and cooling cycles induce thermal expansion and contraction cycles, 
which affect the contact interface and thus the contact resistance of the connectors, which in turn alter their electrical and thermal performances.

During the heating phase, an alternating electrical current is injected into the loop until the reference aluminum conductor reaches the thermal equilibrium at a temperature of $120^{\circ} \mathrm{C}$, the condition attained when injecting an electrical current of about $370 \mathrm{~A}_{\mathrm{RMS}}$. This temperature is superior to $90^{\circ} \mathrm{C}$, the temperature recommended by the conductor' manufacturer, thus accelerating the thermal degradation process.

According to the IEC 61238-1-3:2018 [11], this electrical current must be maintained for $15 \mathrm{~min}$ after attaining the thermal equilibrium. After this time, the current is switched off so that it cools down to ambient temperature with the help of forced ventilation fans, and next, a new heat cycle can start.

HCTs were performed using a high-current $400 \mathrm{~V} / 6 \mathrm{~V}$ variable transformer, supplying the electrical loop as shown in Figure 1a. The rated output of this transformer is $6 \mathrm{~V}_{\mathrm{RMS}}$ and $2.5 \mathrm{kA}_{\mathrm{RMS}}$. A Rogowski coil with a sensitivity of $0.06 \mathrm{mV} / \mathrm{A}$ (500LFxB from PEM, Nottingham, UK) was used to measure the electrical current flowing in the loop. The voltage drop waveforms between the external terminals of the connectors were acquired using a USB-6210 DAQ instrument (National Instruments, Austin, TX, USA) with 8 differential inputs. To transform the resistance of the connectors to $20^{\circ} \mathrm{C}$, T-type thermocouples were used, jointly with a USB TC-08 thermocouple data acquisition module (Omega, Bienne, Switzerland).

\section{The Braunovic Degradation Resistance Model}

This section describes the model used in this paper to describe the degradation of the contact resistance. This model is used in two of the three SoH methods compared in this work. By modelling the degradation of the contact resistance, it is possible to forecast the time evolution of the resistance and, thus, develop SoH methods.

It is known that the resistance of the connectors tends to increase with time, mainly due to the evolution of the contact resistance term. The resistance degradation model analyzed in this paper is based on the increase in the contact resistance with time. As described in the IEC 61238-1-1 standard for medium voltage power connectors [31], the resistance of the connectors evolves with time according to three phases. In the initial phase or the formation phase, after their installation, the resistance of the connectors changes due to the creation of stable constriction surfaces. The next phase is characterized by relative stabilized values of the resistance. In the last phase or accelerated degradation phase, the resistance changes abruptly because the connector is approaching the end of life.

According to Braunovic et al. [33], the temporal evolution of the electrical resistance can be described by a two-parameter degradation model as,

$$
R\left(t, R_{0}, \tau\right)=\frac{R_{0}}{\left(1-\left(\frac{t}{\tau}\right)^{1 / 2}\right)^{2} \times\left(1+\frac{t}{\tau}\right)}
$$

where $R_{0}$ is the initial value of the connector resistance, i.e., the value measured during its installation; $t$ is the actual time measured from the instant of the installation; $\tau$ is the maximal lifetime, corresponding to a vertical asymptote of Equation (2). It is noted that Equation (2) corresponds to a two-parameter $\left(R_{0}, \tau\right)$ model, and these parameters are determined from experimental data.

\section{The Proposed SoH Approach}

This section describes the methods applied to predict the SoH of the power connectors. As explained, three methods are compared to determine the SoH of the connector. They are named linear fitting model (LF-SoH), non-linear fitting model (NLF-SoH) and Markov chain Monte Carlo non-linear model (MCMC-NLF-SoH), and are described in the following subsections. The three methods predict the value of the resistance based on measured past values, as shown in Figure 2. 


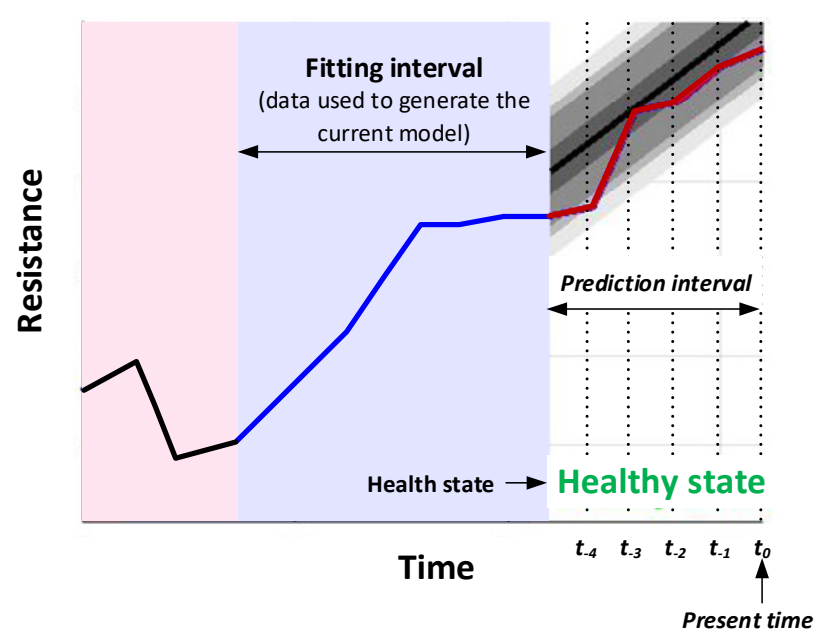

Figure 2. Proposed online SoH prediction strategy showing the 50\%, 90\%, 95\% and 99\% confidence intervals plotted as area bands.

As shown in Figure 2, the algorithm predicts the $\mathrm{SoH}$ of the connector at the present time $t_{0}$, based on the model generated considering the degradation path of the resistance within some pre-established time intervals (fitting interval; blue area in Figure 2). Centered at the present time $t_{0}$, the algorithm takes the past values of the resistance (blue area in Figure 2) and fits the measured values of the resistance to a given equation, which corresponds to a straight line in the LF-SoH approach or to Equation (2) in the NLF-SoH and MCMC-NLF-SoH approaches. Next, the confidence intervals of the regression coefficients are determined, and the resulting regressions are plotted (gray areas in Figure 2). The difference between the NLF-SoH and MCMC-NLF-SoH lies in how the regression coefficients and their confidence intervals are determined. This is detailed in the next subsections. Next, based on the predictions of the model at points $t_{0}, t_{-1}, t_{-2}, t_{-3}$ and $t_{-4}$, the $\mathrm{SoH}$ is predicted according to the strategy proposed in Figure 3.

Figure 3 summarizes the steps of the proposed approach. The first step consists of measuring the present value of the electrical resistance. This is carried out by measuring online the temperature of the connector and the current and voltage drop to determine the resistance by applying Equation (1). Next, the best fitting of the linear degradation model (LF-SoH) or non-linear degradation model given by Equation (2) (NLF-SoH and MCMC-NLF-SoH) is found based on the least-squares algorithm (LF-SoH and NLF-SoH) or the Markov chain Monte Carlo (MCMC) algorithm (MCMC-NLF-SoH), respectively. At this stage, the coefficients of the linear and non-linear models, as well as their confidence intervals, are estimated. Next, a short-term extrapolation based on the obtained regression curves (see the brown curve and the gray areas in Figure 2) is made at points $t_{0}, t_{-1}, t_{-2}$, $t_{-3}$ and $t_{-4}$. The measured values of the resistance at points $t_{0}, t_{-1}, t_{-2}, t_{-3}$ and $t_{-4}$ and those predicted by the regression models are compared to determine the $\mathrm{SoH}$ of the connector, as detailed in the next subsection. 


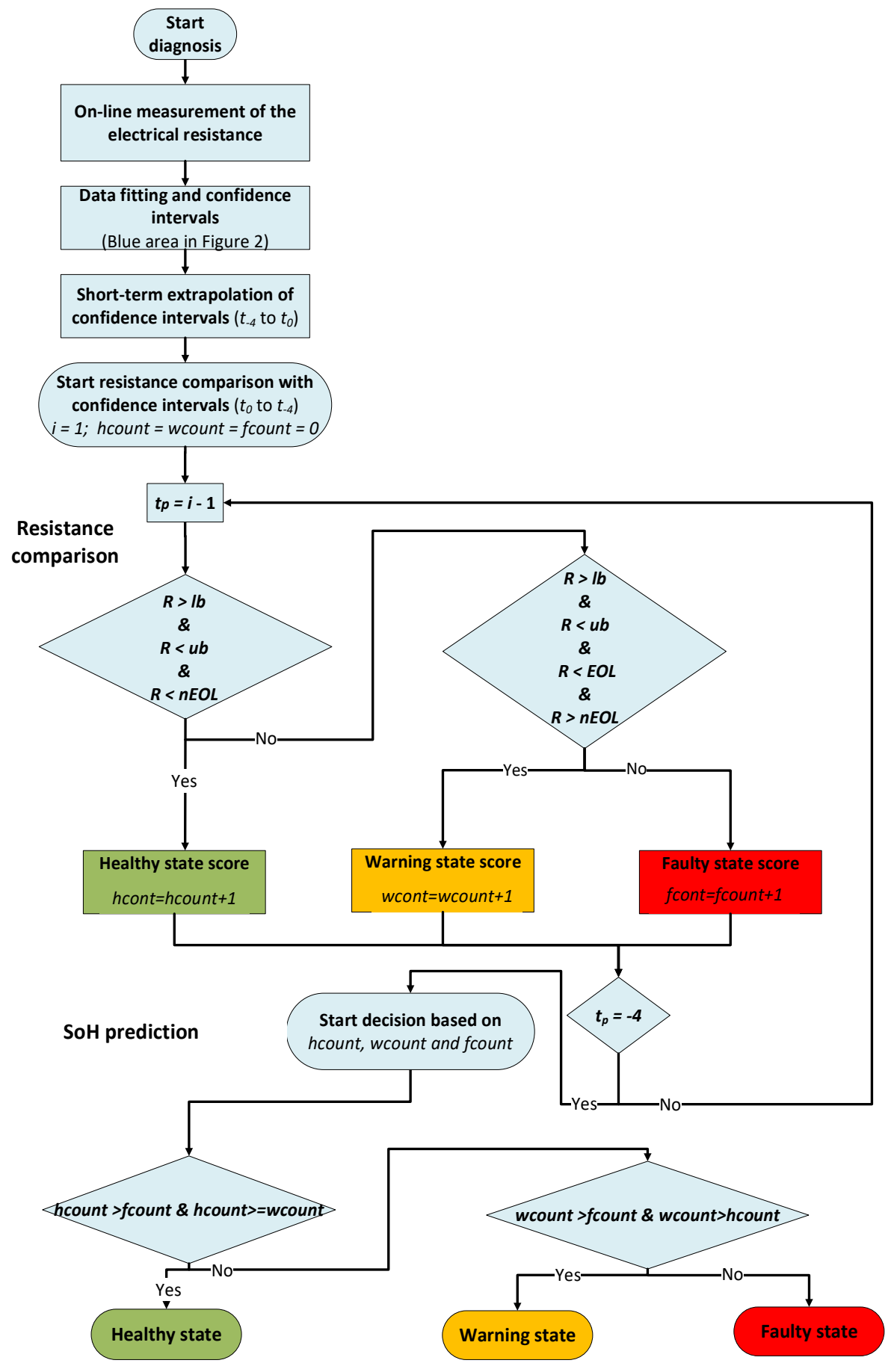

Figure 3. The proposed SoH prediction approach.

\subsection{Resistance Comparison to Determine the $\mathrm{SoH}$}

Figure 3 summarizes the method proposed in this paper to predict the $\mathrm{SoH}$ of the connectors. The LF, NLF and MCMC-NF models are fitted using the measurements obtained during the last $10 \mathrm{~h}$ (fitting interval; blue area in Figure 2) and the mean values of the resistance at points $t_{0}, t_{-1}, t_{-2}, t_{-3}$ and $t_{-4}$ and the lower and upper bounds, $l b$ and $u b$, respectively, are extrapolated by the regression models based on the least-squares values of the model coefficients and their confidence intervals. These are compared against the measured values. The SoH of the connector is predicted from this comparison, which is based on the end-of-life (EOL) and near-EOL (nEOL) of each specific connector. The EOL is defined as $1.4 R_{0}$ [5], whereas the $\mathrm{nEOL}$ is taken as $1.3 R_{0}$. The $\mathrm{nEOL}$ condition 
is used to define a warning condition before reaching the EOL condition since, at this point, the connector must be replaced by a new one to prevent sudden failures and system malfunctioning. This approach allows predicting the SoH of the connectors according to three states, namely healthy, warning and faulty condition, thus facilitating the application of predictive maintenance plans and allowing to schedule maintenance actions.

To categorize the current state of the connector as healthy, warning or faulty, a score is given to each state (hoont, wcount and fcount are the healthy, warning and faulty state counters that provide the final scores) based on the current value of the measured resistance $R$, as detailed in Figure 3, so that the predicted $\mathrm{SoH}$ of the connector is attributed to the most scored state (healthy, warning or faulty).

\subsection{SoH Prediction According to the LF-SoH, NLF-SoH and MCMC-NLF-SoH Methods}

The LF-SoH method predicts the values of the resistance by linearly fitting the past measured values to a straight line. It assumes that the resistance degradation trajectory follows a straight line. By applying the least-squares algorithm, both the coefficients of the linear regression and the confidence intervals of such coefficients are found. The confidence intervals allow confining the predicted values of the resistance within the lower and upper boundaries they define.

Similarly, the NLF-SoH method predicts the values of the resistance by fitting the past measured values to Equation (2), describing the degradation trajectory of the resistance according to Braunovic's model. The coefficients in Equation (2) and their confidence intervals are also found by applying the least-squares algorithm.

The MCMC-NLF-SoH applies the Markov chain Monte Carlo (MCMC) method [34] to find both the values of the coefficients $R_{0}$ and $\tau$ in Equation (2) and their confidence intervals based on the past experimental data. MCMC generates $n$ random samples (3000 samples in this paper $[38,39]$ ) of the coefficients $R_{0}$ and $\tau$ for each simulated time $t(t$ correspond to time points of the last $10 \mathrm{~h}$ ), thus obtaining a matrix of resistances with $n$ rows and $t$ columns. Next, each column is sorted from highest to lowest resistance, and the 99.5th and 0.5th percentiles are calculated (99\% confidence interval). Finally, these values are plotted versus time, as shown in Figure 2. More information about this process can be found in [34].

All codes were programmed by the authors of this work in the MATLAB ${ }^{\circledR}$ environment.

\section{Results}

This section describes the results attained by means of the three methods analyzed in this paper, i.e., the LF-SoH, NLF-SoH and MCMC-NLF-SoH algorithms from the experimental data obtained through the heat cycle tests applied to seven medium-voltage connectors.

\subsection{Results Attained by Applying the LF-SoH Method}

Figure 4 summarizes the results attained with the seven connectors by applying the LF-SoH method every $5 \mathrm{~h}$. It is noted that the green color indicates the healthy state, the orange color the warning or pre-fault state and the red color that the connector has reached the faulty state. Results show that according to the LF algorithm and the rules described in Figure 3, connector \#2 at hour 80, connector \#3 at hour 75, connector \#4 at hour 65, connector \#5 at hour 50 and connector \#6 at hour 25 have reached the fault condition by these time points, so they must be replaced to ensure a safe operation. On the other hand, connectors \#1 and \#7 still do not reach the faulty state at the end of the tests. 


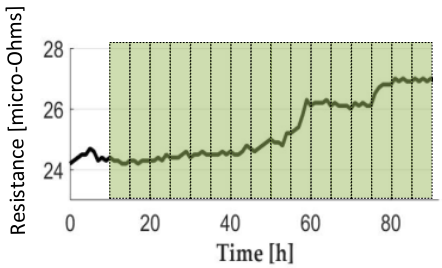

(a)

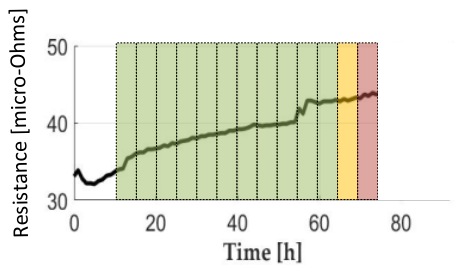

(c)

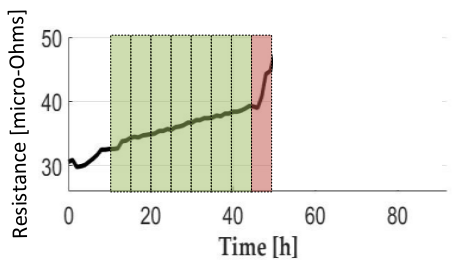

(e)

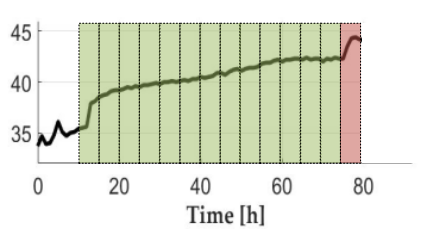

(b)

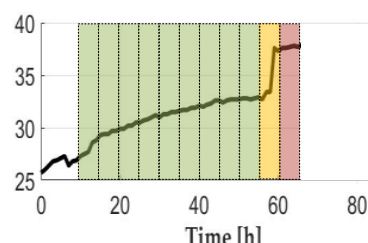

(d)

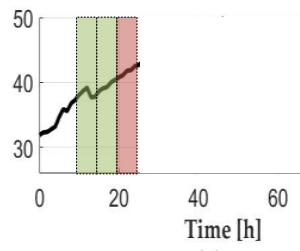

(f)

Healthy state

Warning state

Faulty state

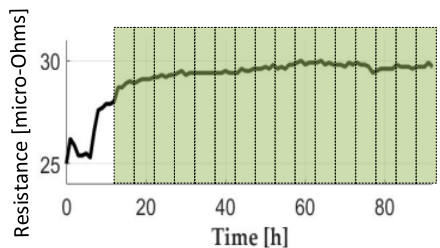

(g)

Figure 4. State of health of the 7 connectors every $5 \mathrm{~h}$ by using the LF-SoH method: (a) Connector $\# 1$, (b) connector \#2, (c) connector \#3, (d) connector \#4, (e) connector \#5, (f) connector \#6 and (g) connector \#7.

Figure 5a,b shows the time evolution of the SoH prediction of connectors \#4 and \#5, respectively. As can be seen in Figure 5, connector \#4 operates under healthy condition at the present times $t_{0}=15 \mathrm{~h}, t_{0}=30 \mathrm{~h}$ and $t_{0}=35 \mathrm{~h}$, whereas connector \#5 shows healthy state operation at the present times $t_{0}=15 \mathrm{~h}, t_{0}=30 \mathrm{~h}$ and $t_{0}=40 \mathrm{~h}$. Finally, both connectors reach the faulty state at $t_{0}=60 \mathrm{~h}$ and $t_{0}=50 \mathrm{~h}$, respectively, due to the important change of their electrical resistances.
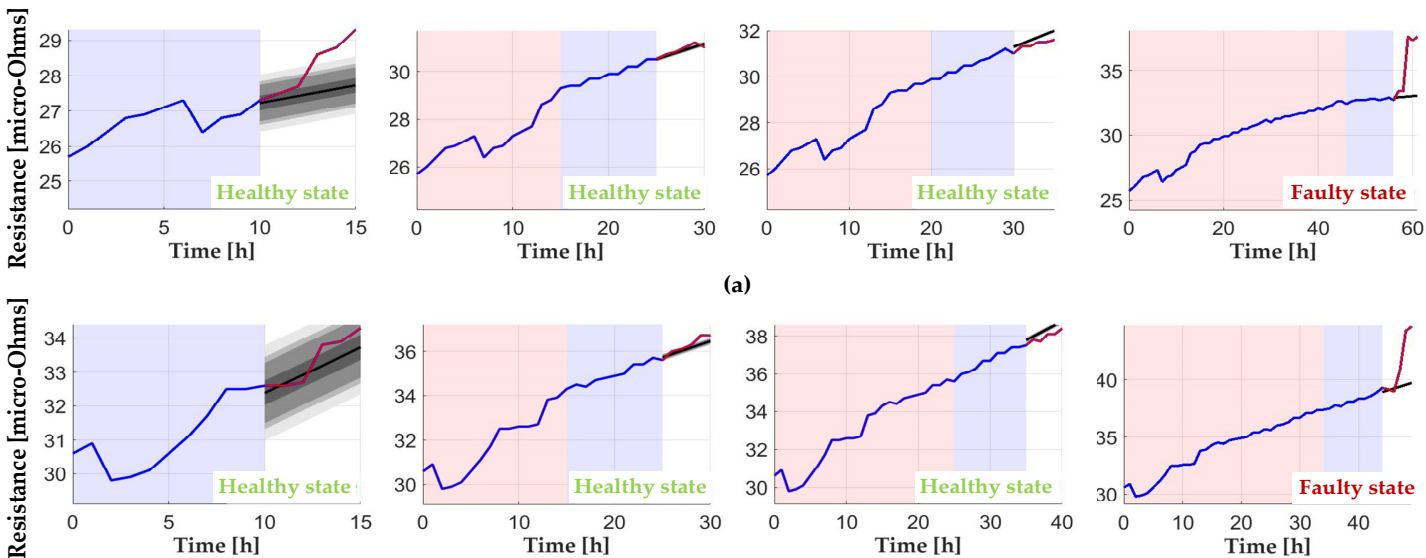

(b)

Figure 5. SoH of connectors \#4 and \#5 predicted by the LF-SoH method at different times. (a) Connector \#4 at present times, $t_{0}=15 \mathrm{~h}, 30 \mathrm{~h}, 35 \mathrm{~h}$ and $60 \mathrm{~h}$. (b) Connector $\# 5$ at $t_{0}=15 \mathrm{~h}, 30 \mathrm{~h}, 40 \mathrm{~h}$ and $50 \mathrm{~h}$. 


\subsection{Results Attained by Applying the NLF-SoH Method}

Figure 6 shows the results obtained with the seven connectors by applying the NLFSoH method every $5 \mathrm{~h}$. These results show that according to the NLF algorithm and the rules described in Figure 3, connector \#2 at hour 80, connector \#3 at hour 70, connector \#4 at hour 60 , connector \#5 at hour 50 and connector \#6 at hour 25 have reached the faulty condition by these time points, so they must be replaced. Similar to the predictions of the LF-SoH method, connectors \#1 and \#7 still do not reach the faulty state at the end of the tests.

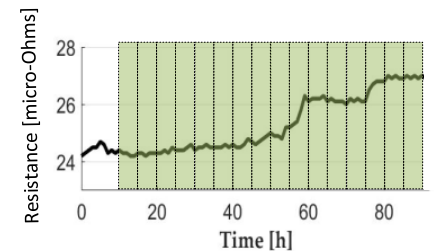

(a)

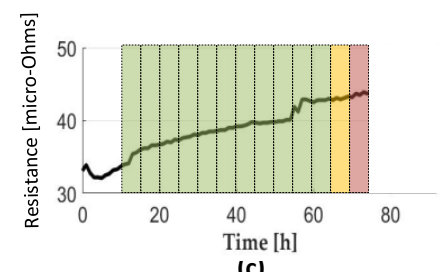

(c)

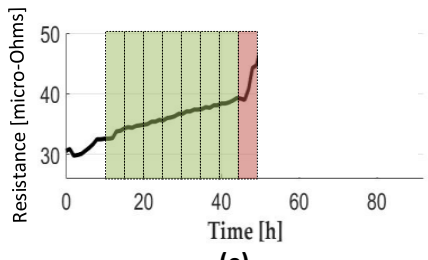

(e)

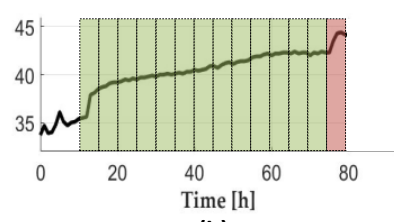

(b)
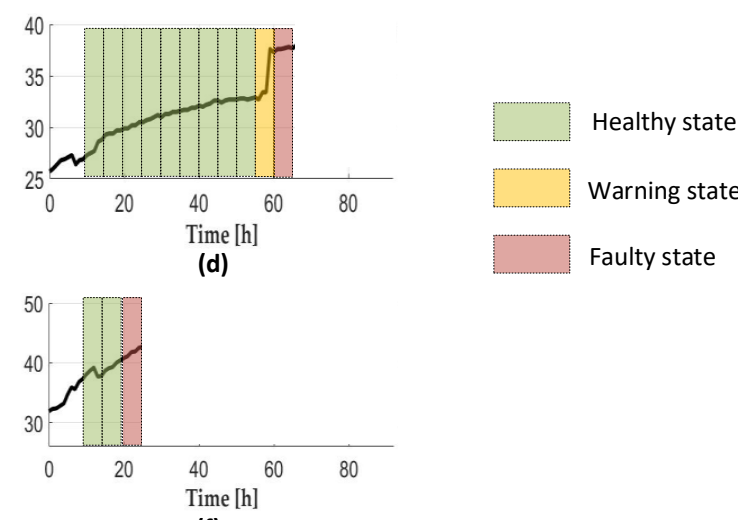

(f)

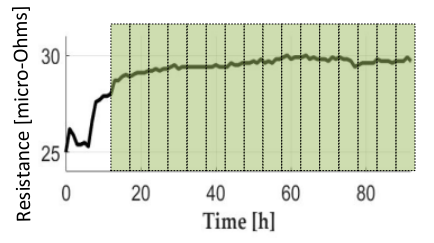

(g)

Figure 6. State of health of the 7 connectors every $5 \mathrm{~h}$ by using the NLF-SoH method: (a) Connector \#1, (b) connector \#2, (c) connector \#3, (d) connector \#4, (e) connector \#5, (f) connector \#6 and (g) connector \#7.

Figure $7 \mathrm{a}, \mathrm{b}$ shows the time evolution of the $\mathrm{SoH}$ prediction of connectors \#4 and \#5, respectively. These figures also show that both connectors experience a sudden change of the resistance increase before $t_{0}=15 \mathrm{~h}$, which is attributed to the initial or formation phase of the resistance, which is quite common at the first hours, so it is reported as a healthy state. Figure $7 \mathrm{a}$ shows that connector $\# 4$ reaches the faulty state at $t_{0}=60 \mathrm{~h}$, whereas connector $\# 5$ at $t_{0}=50 \mathrm{~h}$. 

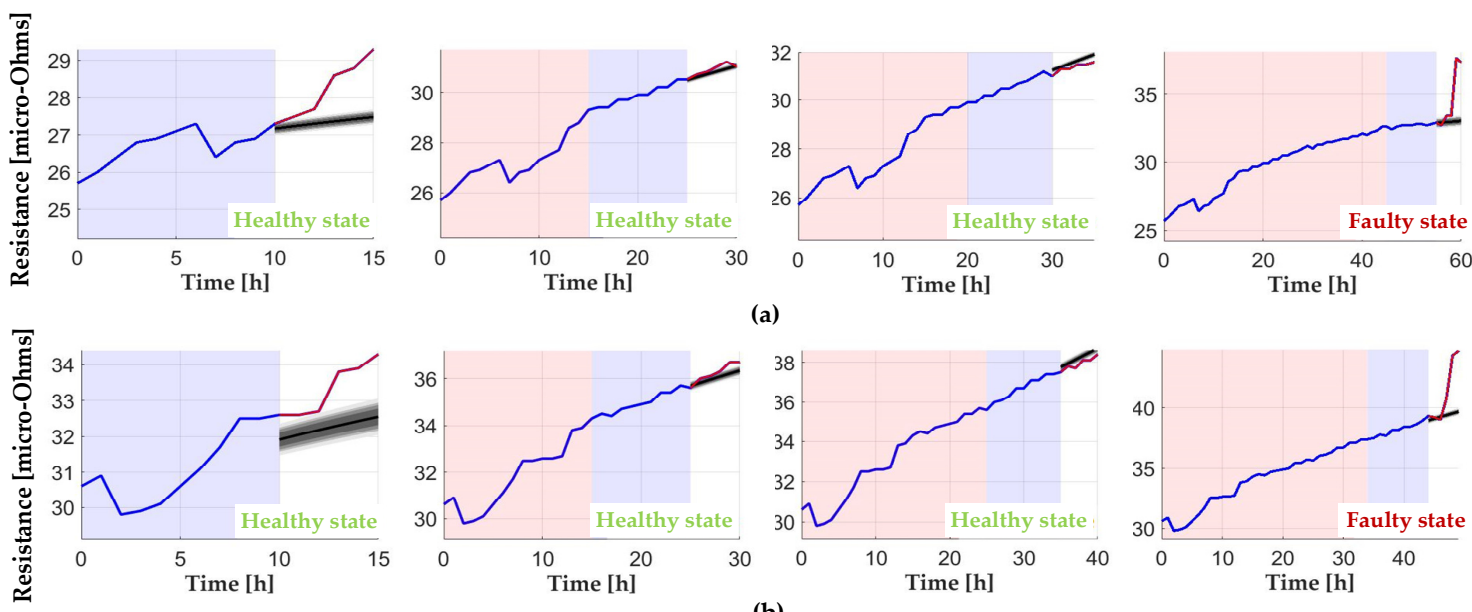

Figure 7. SoH of connectors \#4 and \#5 predicted by the NLF-SoH method at different times. (a) Connector \#4 at present times $t_{0}=15 \mathrm{~h}, 30 \mathrm{~h}, 35 \mathrm{~h}$ and $60 \mathrm{~h}$. (b) Connector \#5 at $t_{0}=15 \mathrm{~h}, 30 \mathrm{~h}, 40 \mathrm{~h}$ and $50 \mathrm{~h}$.

\subsection{Results Attained by Applying the MCMC-NLF-SoH Method}

Figure 8 summarizes the results obtained with the seven connectors by applying the MCMC-NLF-SoH method every $5 \mathrm{~h}$. These results show that according to this algorithm and the rules described in Figure 3, connector \#2 at hour 80, connector \#3 at hour 70, connector $\# 4$ at hour 65 , connector \#5 at hour 50 and connector \#6 at hour 25 have reached the fault condition by these time points. Similar to the predictions of the LF-SoH and NLF-SoH methods, connectors \#1 and \#7 still do not reach the faulty state at the end of the tests.

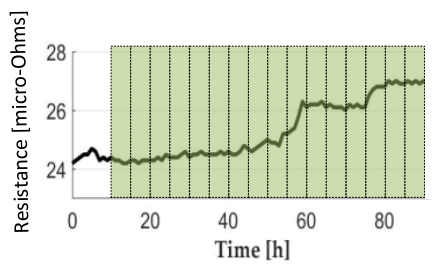

(a)

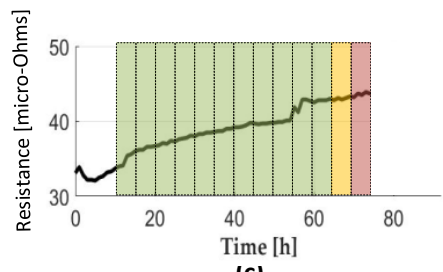

(c)

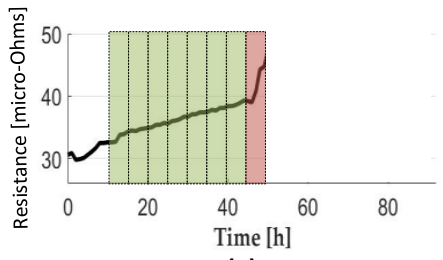

(e)

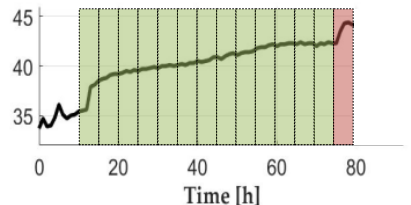

(b)

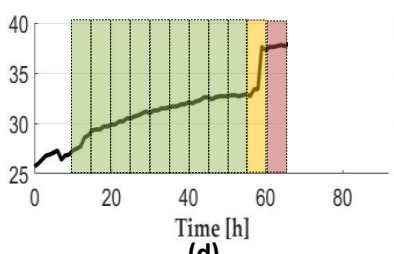

(d)

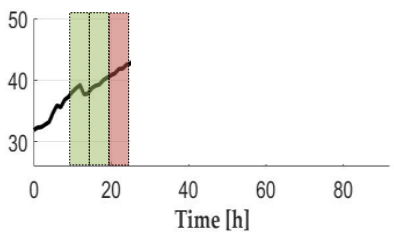

(f)

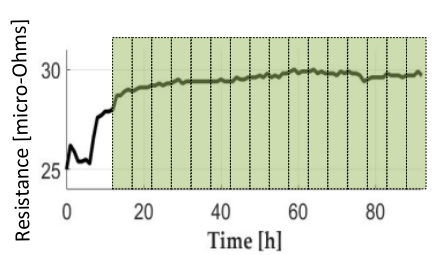

(g)

Figure 8. State of health of the 7 connectors every $5 \mathrm{~h}$ by using the MCMC-NLF-SoH method: (a) Connector \#1, (b) connector \#2, (c) connector \#3, (d) connector \#4, (e) connector \#5, (f) connector \#6 and (g) connector \#7. 
Figure 9a,b shows the time evolution of the SoH prediction of connectors \#4 and \#5, respectively. They indicate that connector \#4 reaches the faulty state at $t_{0}=60 \mathrm{~h}$, whereas connector $\# 5$ reaches the faulty state at $t_{0}=50 \mathrm{~h}$.
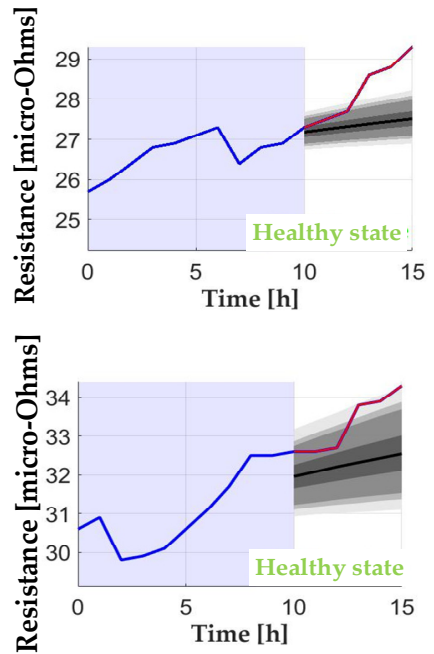

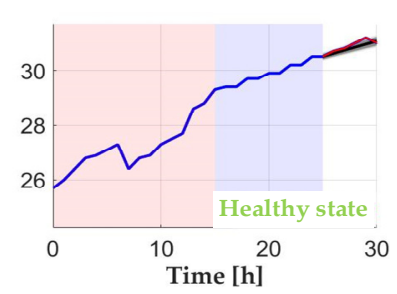

(a)

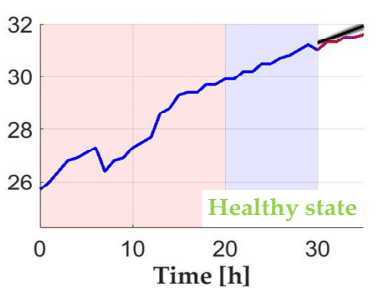

(a)

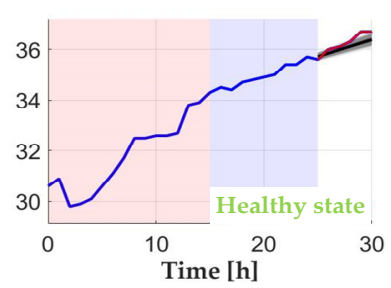

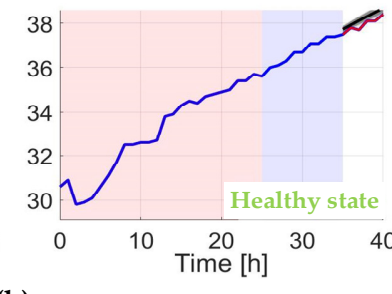

(b)
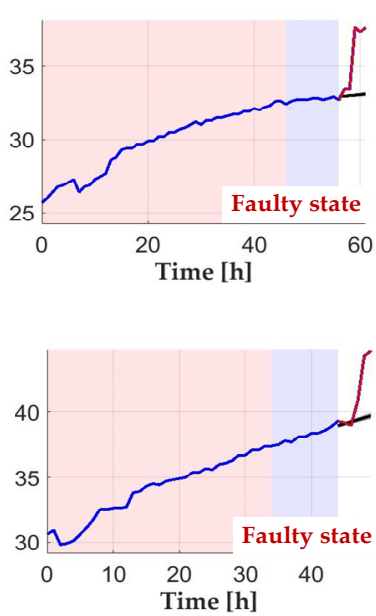

Figure 9. SoH of connectors \#4 and \#5 predicted by the MCMC-NLF-SoH method at different times. (a) Connector \#4 at present times $t_{0}=15 \mathrm{~h}, 30 \mathrm{~h}, 35 \mathrm{~h}$ and $60 \mathrm{~h}$. (b) Connector $\# 5$ at $t_{0}=15 \mathrm{~h}, 30 \mathrm{~h}, 40 \mathrm{~h}$ and $50 \mathrm{~h}$.

\section{Discussion}

The medium-voltage market is very competitive, so in order to reduce costs, mediumvoltage connectors are designed with the minimum material; thus, their outer diameter being quite similar to that of the conductor at which they are connected. Therefore, mediumvoltage connectors are very sensitive to diverse factors, including defects in the conductors or conductors, presence of dirt or oxides, or installation practices, since the crimping process has a great influence. Therefore, the differences among connectors already seen in the previous section are attributed to a combination of these effects, which greatly affect the time to failure.

The first method (linear fitting SoH or LF-SoH) predicts the SoH of a particular connector by comparing the last measured values of the resistance against the predictions determined by a least-squares linear regression model. The other two methods (NLF-SoH and MCMC-NLF-SoH) apply a non-linear model of the degradation trajectory of the contact resistance instead of using a linear degradation model. Whereas NLF-SoH determines the SoH by comparing the last measured values of the resistance against the predictions determined by a least-squares fitting of the Braunovic model, MCMC-NLF-SoH applies the Markov chain Monte Carlo (MCMC) method for this purpose.

Table 1 compares the results attained with the three analyzed SoH prediction methods to better understand their behavior.

The results presented in Table 1 show a similar performance of the three methods since they predict the healthy, warning and faulty conditions at the same time instants.

Table 2 shows the average time required by the three methods to predict the $\mathrm{SoH}$ of the connectors.

From the results summarized in Tables 1 and 2, it can be concluded that the three methods exhibit comparable results and that the LF-SoH method, due to its simplicity, is the one requiring lower computational requirements with a similar performance than the NLF-SoH and MCMC-NLF-SoH methods. It is also noted that the MCMC-NLF-SoH algorithm is the one requiring more computational resources. 
Table 1. State of health of the 7 connectors every $5 \mathrm{~h}$ according to the LF/NLF/MCMC-NLF-SoH methods.

\begin{tabular}{|c|c|c|c|c|c|c|c|}
\hline \multirow{2}{*}{ Present Time, $t_{0}(\mathrm{~h})$} & \multicolumn{7}{|c|}{ Connector's Resistance (Micro-Ohms) } \\
\hline & $\# 1$ & $\# 2$ & $\# 3$ & $\# 4$ & \#5 & \#6 & \#7 \\
\hline 15 & $\mathrm{H} / \mathrm{H} / \mathrm{H}$ & $\mathrm{H} / \mathrm{H} / \mathrm{H}$ & $\mathrm{H} / \mathrm{H} / \mathrm{H}$ & $\mathrm{H} / \mathrm{H} / \mathrm{H}$ & $\mathrm{H} / \mathrm{H} / \mathrm{H}$ & $\mathrm{H} / \mathrm{H} / \mathrm{H}$ & $\mathrm{H} / \mathrm{H} / \mathrm{H}$ \\
\hline 20 & $\mathrm{H} / \mathrm{H} / \mathrm{H}$ & $\mathrm{H} / \mathrm{H} / \mathrm{H}$ & $\mathrm{H} / \mathrm{H} / \mathrm{H}$ & $\mathrm{H} / \mathrm{H} / \mathrm{H}$ & $\mathrm{H} / \mathrm{H} / \mathrm{H}$ & $\mathrm{H} / \mathrm{H} / \mathrm{H}$ & $\mathrm{H} / \mathrm{H} / \mathrm{H}$ \\
\hline 25 & $\mathrm{H} / \mathrm{H} / \mathrm{H}$ & $\mathrm{H} / \mathrm{H} / \mathrm{H}$ & $\mathrm{H} / \mathrm{H} / \mathrm{H}$ & $\mathrm{H} / \mathrm{H} / \mathrm{H}$ & $\mathrm{H} / \mathrm{H} / \mathrm{H}$ & $\mathrm{F} / \mathrm{F} / \mathrm{F}$ & $\mathrm{H} / \mathrm{H} / \mathrm{H}$ \\
\hline 30 & $\mathrm{H} / \mathrm{H} / \mathrm{H}$ & $\mathrm{H} / \mathrm{H} / \mathrm{H}$ & $\mathrm{H} / \mathrm{H} / \mathrm{H}$ & $\mathrm{H} / \mathrm{H} / \mathrm{H}$ & $\mathrm{H} / \mathrm{H} / \mathrm{H}$ & & $\mathrm{H} / \mathrm{H} / \mathrm{H}$ \\
\hline 35 & $\mathrm{H} / \mathrm{H} / \mathrm{H}$ & $\mathrm{H} / \mathrm{H} / \mathrm{H}$ & $\mathrm{H} / \mathrm{H} / \mathrm{H}$ & $\mathrm{H} / \mathrm{H} / \mathrm{H}$ & $\mathrm{H} / \mathrm{H} / \mathrm{H}$ & & $\mathrm{H} / \mathrm{H} / \mathrm{H}$ \\
\hline 40 & $\mathrm{H} / \mathrm{H} / \mathrm{H}$ & $\mathrm{H} / \mathrm{H} / \mathrm{H}$ & $\mathrm{H} / \mathrm{H} / \mathrm{H}$ & $\mathrm{H} / \mathrm{H} / \mathrm{H}$ & $\mathrm{H} / \mathrm{H} / \mathrm{H}$ & & $\mathrm{H} / \mathrm{H} / \mathrm{H}$ \\
\hline 45 & $\mathrm{H} / \mathrm{H} / \mathrm{H}$ & $\mathrm{H} / \mathrm{H} / \mathrm{H}$ & $\mathrm{H} / \mathrm{H} / \mathrm{H}$ & $\mathrm{H} / \mathrm{H} / \mathrm{H}$ & $\mathrm{H} / \mathrm{H} / \mathrm{H}$ & & $\mathrm{H} / \mathrm{H} / \mathrm{H}$ \\
\hline 50 & $\mathrm{H} / \mathrm{H} / \mathrm{H}$ & $\mathrm{H} / \mathrm{H} / \mathrm{H}$ & $\mathrm{H} / \mathrm{H} / \mathrm{H}$ & $\mathrm{H} / \mathrm{H} / \mathrm{H}$ & $\mathrm{F} / \mathrm{F} / \mathrm{F}$ & & $\mathrm{H} / \mathrm{H} / \mathrm{H}$ \\
\hline 55 & $\mathrm{H} / \mathrm{H} / \mathrm{H}$ & $\mathrm{H} / \mathrm{H} / \mathrm{H}$ & $\mathrm{H} / \mathrm{H} / \mathrm{H}$ & $\mathrm{H} / \mathrm{H} / \mathrm{H}$ & & & $\mathrm{H} / \mathrm{H} / \mathrm{H}$ \\
\hline 60 & $\mathrm{H} / \mathrm{H} / \mathrm{H}$ & $\mathrm{H} / \mathrm{H} / \mathrm{H}$ & $\mathrm{H} / \mathrm{H} / \mathrm{H}$ & $\mathrm{W} / \mathrm{W} / \mathrm{W}$ & & & $\mathrm{H} / \mathrm{H} / \mathrm{H}$ \\
\hline 65 & $\mathrm{H} / \mathrm{H} / \mathrm{H}$ & $\mathrm{H} / \mathrm{H} / \mathrm{H}$ & $\mathrm{H} / \mathrm{H} / \mathrm{H}$ & $\mathrm{F} / \mathrm{F} / \mathrm{F}$ & & & $\mathrm{H} / \mathrm{H} / \mathrm{H}$ \\
\hline 70 & $\mathrm{H} / \mathrm{H} / \mathrm{H}$ & $\mathrm{H} / \mathrm{H} / \mathrm{H}$ & $\mathrm{W} / \mathrm{W} / \mathrm{W}$ & & & & $\mathrm{H} / \mathrm{H} / \mathrm{H}$ \\
\hline 75 & $\mathrm{H} / \mathrm{H} / \mathrm{H}$ & $\mathrm{H} / \mathrm{H} / \mathrm{H}$ & $\mathrm{F} / \mathrm{F} / \mathrm{F}$ & & & & $\mathrm{H} / \mathrm{H} / \mathrm{H}$ \\
\hline 80 & $\mathrm{H} / \mathrm{H} / \mathrm{H}$ & $\mathrm{F} / \mathrm{F} / \mathrm{F}$ & & & & & $\mathrm{H} / \mathrm{H} / \mathrm{H}$ \\
\hline 85 & $\mathrm{H} / \mathrm{H} / \mathrm{H}$ & & & & & & $\mathrm{H} / \mathrm{H} / \mathrm{H}$ \\
\hline 90 & $\mathrm{H} / \mathrm{H} / \mathrm{H}$ & & & & & & $\mathrm{H} / \mathrm{H} / \mathrm{H}$ \\
\hline
\end{tabular}

Table 2. The average computation time required by the three methods using an Intel ${ }^{\circledR}$ Core (TM) i7-8750H CPU @2.20 GHz.

\begin{tabular}{cc}
\hline Method & Computation Time \\
\hline LF-SoH & $6 \mathrm{~ms}$ \\
NLF-SoH & $50 \mathrm{~ms}$ \\
MCMC-NLF-SoH & $1200 \mathrm{~ms}$ \\
\hline
\end{tabular}

\section{Conclusions}

Power connectors are critical elements in power lines and networks, so their failure can lead to important consequences, including power outages, safety-related issues and economic losses. Thus, there is an increasing need to continuously monitor their performance in order to ensure high reliability by anticipating the fault condition before occurrence. With the development of low-cost sensors and wireless communications systems compatible with the Internet of Things, this topic is receiving much attention and interest because these developments facilitate the application of predictive maintenance approaches. Despite the enormous implications of future developments in this area, there is a scarcity of works dealing with this topic applied to power connectors, this paper contributing to this field.

This work has presented, analyzed and compared the behavior of three methods for an online determination of the SoH of power connectors, namely LF-SoH, NLF-SoH and MCMC-NLF-SoH. These methods were selected based on simplicity, low computational requirements and adaptation to the particular characteristics and behavior of each connector. They rely on monitoring the degradation trajectory of the electrical resistance of the connectors, thus detecting changes that allow anticipating the fault before occurrence so that predictive maintenance actions can be applied. The resistance is used as an indicator of their SoH because it is known that degradation of the connectors' thermal end electrical behavior is associated with changes of this magnitude. To obtain reliable and realistic data, seven medium voltage connectors were subjected to accelerated heat cycle tests. The electrical resistance of the seven connectors was monitored by measuring the temperature, electrical current and voltage drop across the terminal points of the connectors.

From the experimental validation carried out in this research work, it is concluded that although the three methods show comparable results, the LF-SoH method is the fastest one due to its simplicity, whereas the MCMC-NLF-SoH algorithm is the one requiring more computational resources. 
The results presented show the fast and accurate response and reduced computational burden of the three assessed methods and their potential to predict the $\mathrm{SoH}$ of power connectors. This approach can also be applied to many other power devices and components.

The results presented in this work are intended to assess the feasibility and potential to apply the proposed method to predict the $\mathrm{SoH}$ of substation connectors instead of medium voltage connectors. For future work, this requires including an energy harvesting unit, several sensors (temperature, voltage drop and current) and wireless communications [4].

Author Contributions: Conceptualization, J.-R.R. and J.M.; methodology, J.-R.R.; formal analysis, M.M.-E. and J.M.; investigation, J.-R.R. and J.M.; writing-original draft preparation, J.-R.R.; writingreview and editing, M.M.-E. and J.M. All authors have read and agreed to the published version of the manuscript.

Funding: This research was partially funded by the Ministerio de Ciencia, Innovación y Universidades de España, grant number RTC-2017-6297-3 and by the Generalitat de Catalunya, grant number 2017 SGR 967.

Conflicts of Interest: The authors declare no conflict of interest.

\section{References}

1. Capelli, F.; Riba, J.-R.; Sanllehí, J. Finite element analysis to predict temperature rise tests in high-capacity substation connectors. IET Gener. Transm. Distrib. 2017, 11, 2283-2291. [CrossRef]

2. CEER. 6TH CEER Benchmarking Report on the Quality of Electricity and Gas Supply; CEER: Brussels, Belgium, 2016.

3. de Paulis, F.; Olivieri, C.; Orlandi, A.; Giannuzzi, G. Detectability of Degraded Joint Discontinuities in HV Power Lines Through TDR-Like Remote Monitoring. IEEE Trans. Instrum. Meas. 2016, 65, 2725-2733. [CrossRef]

4. Kadechkar, A.; Riba, J.R.; Moreno-Eguilaz, M.; Perez, J. SmartConnector: A Self-Powered IoT Solution to Ease Predictive Maintenance in Substations. IEEE Sens. J. 2020, 20, 11632-11641. [CrossRef]

5. Gómez-Pau, Á.; Riba, J.-R.; Moreno-Eguilaz, M. Time Series RUL Estimation of Medium Voltage Connectors to Ease Predictive Maintenance Plans. Appl. Sci. 2020, 10, 9041. [CrossRef]

6. ANSI/NEMA. ANSI/NEMA CC1 Electric Power Connection for Substation; ANSI/NEMA: Rosslyn, VA, USA, 2009.

7. Riba, J.-R.; Mancini, A.-G.; Abomailek, C.; Capelli, F. 3D-FEM-Based Model to Predict the Electrical Constriction Resistance of Compressed Contacts. Measurement 2018, 114, 44-50. [CrossRef]

8. Riba, J.-R.; Martínez, J.; Moreno-Eguilaz, M.; Capelli, F. Characterizing the temperature dependence of the contact resistance in substation connectors. Sens. Actuators A Phys. 2021, 327, 112732. [CrossRef]

9. Capelli, F.; Riba, J.; Ruperez, E.; Sanllehi, J. A Genetic-Algorithm-Optimized Fractal Model to Predict the Constriction Resistance From Surface Roughness Measurements. IEEE Trans. Instrum. Meas. 2017, 66, 2437-2447. [CrossRef]

10. Shen, Q.; Lv, K.; Liu, G.; Qiu, J. Dynamic Performance of Electrical Connector Contact Resistance and Intermittent Fault Under Vibration. IEEE Trans. Compon. Packag. Manuf. Technol. 2018, 8, 216-225. [CrossRef]

11. International Electrotechnical Commission. Compression and Mechanical Connectors For Power Cables-Part 1-3: Test Methods and Requirements for Compression and Mechanical Connectors for Power Cables for Rated Voltages Above $1 \mathrm{kV}$ (Um=1,2 kV) Up to $30 \mathrm{kV}$ (Um $=36$ kV) Tested on 2018; International Electrotechnical Commission: Geneva, Switzerland, 2018; pp. 1-89.

12. Qian, P.; Hong, L.; Chen, W.; Qian, Y.; Wang, Z.; Yao, H. Optimization of the Accelerated Degradation Test Plan for Electrical Connector Contact Pairs Based on a Nonlinear Wiener Process. Math. Probl. Eng. 2020, 2020, 1-16. [CrossRef]

13. Tadeusiewicz, M.; Hałgas, S. Parametric Fault Diagnosis of Very High-Frequency Circuits Containing Distributed Parameter Transmission Lines. Electronics 2021, 10, 550. [CrossRef]

14. Xu, Q.; Huang, H.; Zhou, C.; Zhang, X. Research on Real-Time Infrared Image Fault Detection of Substation High-Voltage Lead Connectors Based on Improved YOLOv3 Network. Electronics 2021, 10, 544. [CrossRef]

15. Martinez, D.; Henao, H.; Capolino, G.A. Overview of Condition Monitoring Systems for Power Distribution Grids. In Proceedings of the 2019 IEEE 12th International Symposium on Diagnostics for Electrical Machines, Power Electronics and Drives, Toulouse, France, 27-30 August 2019; pp. 160-166.

16. Van Deursen, A.; Wouters, P.; Steennis, F. Corrosion in low-voltage distribution networks and perspectives for online condition monitoring. IEEE Trans. Power Deliv. 2019, 34, 1423-1431. [CrossRef]

17. Seo, J.; Ma, H.; Saha, T.K. On savitzky-golay filtering for online condition monitoring of transformer on-load tap changer. IEEE Trans. Power Deliv. 2018, 33, 1689-1698. [CrossRef]

18. Razi-Kazemi, A.A.; Shariatnasab, M. A New Approach on Prioritization of the Circuit Breakers for Installation of Online Monitoring Systems. IEEE Trans. Power Deliv. 2019, 34, 1569-1577. [CrossRef]

19. Joseph, J.; Krishnan, S.T. Development of Severity and Location Indices Based Condition Monitoring Scheme for Underground Cables by Impedance Spectroscopy. IEEE Trans. Power Deliv. 2020, 36, 533-543. [CrossRef] 
20. Wei, Y.; Wu, D.; Terpenny, J. Robust Incipient Fault Detection of Complex Systems Using Data Fusion. IEEE Trans. Instrum. Meas. 2020, 69, 9526-9534. [CrossRef]

21. Yang, F.; Du, L.; Yu, H.; Huang, P. Magnetic and Electric Energy Harvesting Technologies in Power Grids: A Review. Sensors 2020, 20, 1496. [CrossRef]

22. Susinni, G.; Rizzo, S.A.; Iannuzzo, F. Two Decades of Condition Monitoring Methods for Power Devices. Electronics 2021, 10, 683. [CrossRef]

23. Furse, C.M.; Kafal, M.; Razzaghi, R.; Shin, Y.J. Fault Diagnosis for Electrical Systems and Power Networks: A Review. IEEE Sens. J. 2021, 21, 888-906. [CrossRef]

24. Huang, J.; Fukuda, T.; Matsuno, T. Model-based intelligent fault detection and diagnosis for mating electric connectors in robotic wiring harness assembly systems. IEEE/ASME Trans. Mechatron. 2008, 13, 86-94. [CrossRef]

25. Chen, L.; Xu, L.; Zhou, Y. Novel Approach for Lithium-Ion Battery On-Line Remaining Useful Life Prediction Based on Permutation Entropy. Energies 2018, 11, 820. [CrossRef]

26. Ramezani, S.; Moini, A.; Riahi, M. Prognostics and Health Management in Machinery: A Review of Methodologies for RUL prediction and Roadmap; University of Hormozgan: Bandar Abbas, Iran, 2019; Volume 6.

27. Hu, X.; Xu, L.; Lin, X.; Pecht, M. Battery Lifetime Prognostics. Joule 2020, 4, 310-346. [CrossRef]

28. Severson, K.A.; Attia, P.M.; Jin, N.; Perkins, N.; Jiang, B.; Yang, Z.; Chen, M.H.; Aykol, M.; Herring, P.K.; Fraggedakis, D.; et al. Data-driven prediction of battery cycle life before capacity degradation. Nat. Energy 2019, 4, 383-391. [CrossRef]

29. Zhu, J.J.; Yang, Z.C. Thermo-elasto-plastic stress and strain analysis and life prediction of gas turbine blade. In Proceedings of the 2010 International Conference on Measuring Technology and Mechatronics Automation, Changsha, China, 13-14 March 2020; Volume 3, pp. 1019-1022.

30. International Electrotechnical Commission. 2017 Estimation of the Reliability of Electrical Connectors; International Electrotechnical Commission: Geneva, Switzerland, 2017; pp. 1-55.

31. IEC. 2018 Compression and Mechanical Connectors for Power Cables-Part 1-1: Test Methods and Requirements for Compression and Mechanical Connectors for Power Cables for Rated Voltages Up to $1 \mathrm{kV}(U \mathrm{~m}=1,2 \mathrm{kV})$ Tested on Non-Insulated Conductors; IEC: Geneva, Switzerland, 2018; p. 81.

32. Tanwar, M.; Raghavan, N. Lubrication oil degradation trajectory prognosis with ARIMA and bayesian models. In Proceedings of the 2019 International Conference on Sensing, Diagnostics, Prognostics, and Control, Beijing, China, 17 August 2020; pp. 606-611.

33. Braunovic, M.; Izmailov, V.V.; Novoselova, M.V. A model for life time evaluation of closed electrical contacts. Electr. Contacts Proc. Annu. Holm Conf. Electr. Contacts 2005, 2005, 217-223.

34. Martinez, J.; Gomez-Pau, A.; Riba, J.-R.; Moreno-Eguilaz, M. On-Line Health Condition Monitoring of Power Connectors Focused on Predictive Maintenance. IEEE Trans. Power Deliv. 2020, 99, 1. [CrossRef]

35. Liu, H.; Claeys, T.; Pissoort, D.; Vandenbosch, G.A.E. Prediction of Capacitor's Accelerated Ageing Based on Advanced Measurements and Deep Neural Network Techniques. IEEE Trans. Instrum. Meas. 2020, 69, 9019-9027. [CrossRef]

36. Kang, H.; Shim, T.; Kim, S.H.; Jeong, S.; An, Y.Y. A Tesbed for Experimenting IoT Platform on High-Voltage Customer Electrical Installation. In Proceedings of the 9th International Conference on Information and Communication Technology Convergence: ICT Convergence Powered by Smart Intelligence, Jeju, Korea, 17-19 October 2018; pp. 1301-1304.

37. Hsu, T.S.; Chiu, H.C.; Yang, Y.C.; Tseng, C.Y.; Lin, M.J.; Wang, J.C.; Jiang, J.A. An IoT-based Sag Monitoring System for Overhead Transmission Lines. In Proceedings of the 2019 IEEE PES GTD Grand International Conference and Exposition Asia, GTD Asia 2019, Bangkok, Thailand, 20-23 March 2019; pp. 515-519.

38. Dzhamtyrova, R.; Kalnishkan, Y. Competitive Online Generalised Linear Regression with Multidimensional Outputs. In Proceedings of the Institute of Electrical and Electronics Engineers (IEEE), Budapest, Hungary, 14-19 July 2019 ; pp. 1-8.

39. Aghaei, V.T.; Agababaoglu, A.; Onat, A.; Yildirim, S. Bayesian learning for policy search in trajectory control of a planar manipulator. In Proceedings of the 2019 IEEE 9th Annual Computing and Communication Workshop and Conference, Las Vegas, NV, USA, 7-9 January 2019; pp. 240-246. 